\title{
El pragmatismo en la historia inicial de la terapia ocupacional
}

\section{O pragmatismo na história inicial da terapia ocupacional}

\section{Pragmatism in the initial history of occupational therapy}

\author{
Rodolfo Morrison ${ }^{\mathrm{a}}$ (iD \\ ${ }^{a}$ Universidad de Chile, Santiago de Chile, Chile
}

Cómo citar: Morrison, R. (2021). El pragmatismo en la historia inicial de la terapia ocupacional. Cadernos Brasileiros de Terapia Ocupacional, 29, e2147. https://doi.org/10.1590/2526-8910.ctoARF2147

\begin{abstract}
$\underline{\text { Resumen }}$
Cómo movimiento filosófico y fundamento epistemológico de la terapia ocupacional, el pragmatismo es citado en escasos textos al inicio de la profesión. Es por lo anterior, que en este artículo se busca describir las influencias de la filosofía pragmatista en los comienzos de la terapia ocupacional en Estados Unidos de América. Para ello, se centra en dos aspectos: 1. las relaciones de los/as pragmatistas y la primera generación de terapeutas ocupacionales; y 2. el desarrollo teórico de la terapia ocupacional y el pragmatismo, donde se destacan tres aspectos: i) el holismo en la profesión, ii) la significación de la subjetividad de las personas y iii) el pragmatismo de Meyer. Se concluye cómo el pragmatismo, a pesar de no ser una filosofía ampliamente divulgada en la profesión, presenta antecedentes contundentes respecto a su importancia en sus inicios. Finalmente, se plantean algunos desafíos y posibilidades relacionadas con los aportes que el pragmatismo podría tener para la disciplina hoy en día.
\end{abstract}

Palabras-clave: Filosofía/Conocimiento, Historia/Terapia Ocupacional, Epistemología.

\section{$\underline{\text { Resumo }}$}

Como movimento filosófico e fundamento epistemológico da terapia ocupacional, o pragmatismo é citado em poucos textos no início da profissáo. Por esse motivo, este artigo busca descrever as influências da filosofia pragmatista para o início da terapia ocupacional nos Estados Unidos de América. Para isso, concentra-se em dois aspetos: 1. as relaçôes dos/as pragmatistas e a primeira geração de terapeutas ocupacionais; e 2. o desenvolvimento teórico da terapia ocupacional e do pragmatismo, destacando-se três aspetos: i) o holismo na profissão, ii) o significado da subjetividade das pessoas e iii) o pragmatismo de Meyer. Conclui-se como o pragmatismo, apesar de não ser uma filosofia amplamente disseminada na terapia 
ocupacional, apresenta fortes antecedentes no que se refere à importância para o início da profissão. Finalmente, se consideram alguns desafios e possibilidades relacionados às contribuiçóes que o pragmatismo poderia ter para a disciplina atualmente.

Palavras-chave: Filosofia/Conhecimento, História/Terapia Ocupacional, Epistemologia.

\begin{abstract}
$\underline{\text { Abstract }}$
As a philosophical movement and epistemological base of occupational therapy, pragmatism is cited in few texts at the beginning of the profession. For this reason, this article seeks to describe the influences of pragmatist philosophy at the beginning of occupational therapy in the United States of America. To do this, it focuses on two aspects: 1. the relationships of the pragmatists and the first generation of occupational therapists; and 2. the theoretical development of occupational therapy and pragmatism, where three aspects stand out: i) holism in the profession, ii) the significance of people's subjectivity and iii) Meyer's pragmatism. It is concluded how pragmatism, despite not being a widely disseminated philosophy in the profession, presents a strong background regarding its importance in its beginnings. Finally, there are some challenges and possibilities related to the contributions that pragmatism could have for the discipline today.
\end{abstract}

Keywords: Philosophy/Knowledge, History/Occupational Therapy, Epistemology.

\title{
Introducción
}

Una forma de comprender al pragmatismo es como una corriente filosófica norteamericana que propone la imposibilidad de separar la teoría de la práctica. Además, busca plantear un camino para el análisis, la comprensión y el cuestionamiento de "la verdad". Entiende la verdad como un constructo en permanente transformación y que incide en nuestra realidad a través de sus repercusiones prácticas en nuestra vida. También, propone que el pensamiento provee oportunidades únicas para transformar la realidad y que su dirección debería ser siempre hacia la mejora de las situaciones, teorías, investigaciones, condiciones sociales, etc. con las que las personas vivimos, es decir, el pensamiento debe guiar la acción a través de un proceso crítico de reflexiónacción (Morrison, 2017).

Esta filosofía es considerada como un pilar estructural de la terapia ocupacional aunque poco reconocida (Breines, 1986; Hooper \& Wood, 2002; Ikiugu, 2001; Morrison, 2017; Reed, 2017, 2018). La ausencia por muchos años del pragmatismo en la profesión no tiene que ver, solamente, con una cuestión contemporánea. No todos/as los/as fundadores/as de la terapia ocupacional citaron de forma explícita al pragmatismo en sus documentos (Breines, 1986; Slagle, 1936; Tracy, 1910) fueron dos referentes que sí citaron a ragmatistas en sus publicaciones. De forma particular, se refirieron a Jane Addams, William James y John Dewey.

Por otro lado, las principales influencias y contextos teóricos y políticos que se han documentado en los comienzos de la disciplina en los Estados Unidos de América son: 
los conocidos tratamiento moral y movimientos de artes y oficios (Gordon, 2009; Kielhofner, 2009; Levine, 1987); el movimiento de higiene mental (Breines, 1986); el movimiento de los asentamientos sociales, donde se destaca la fundación de la Hull House por parte de Jane Addams (Quiroga, 1995); el clima politico-democrático-liberal que provocó grandes reformas políticas en Chicago (Addams, 1935/2004); y, en menor correspondencia, el movimiento Emmanuel, de tinte religioso-cristiano cuyo desarrollo se acentuó en Baltimore, especialmente en el Hospital Heppard-Pratt donde trabajó William R. Dunton (y que también habría impactado en Herbert J. Hall) (Gordon, 2002). Lo anterior, sumado a la Primera y Segunda Guerra Mundial (Kielhofner, 2009), más la segunda ola del feminismo (Morrison, 2014, 2016a) habría facilitado la constitución de la profesión y la extensión de su desarrollo teórico.

Los elementos referidos anteriormente permitieron que las y los fundadores se congregasen bajo la idea central de que la ocupación constituye un potencial preventivo, curativo y restaurador de funciones y habilidades $y$, por sobre todo, del bienestar y la salud de las personas (Morrison, 2014).

Esta máxima fue compartida tanto por pragmatistas como Addams, James o Dewey, como también por terapeutas ocupacionales como Slagle, Tracy y Johnson (Morrison, 2017). Esto se habría gestado así, pues los intercambios constantes entre la primera generación de terapeutas ocupacionales y los/as pragmatista clásicos/as eran constantes.

Es por lo anterior, que en este artículo se busca describir las influencias de la filosofía pragmatista en los comienzos de la terapia ocupacional en los Estados Unidos de América, a través de dos momentos. El primero da cuenta de los vínculos entre pragmatistas y terapeutas ocupacionales; y el segundo, sobre la integración de algunas ideas pragmatistas en diferentes aspectos del desarrollo teórico de la disciplina.

\section{Los/as Pragmatistas y la Primera Generación de Terapeutas Ocupacionales}

Emil Gustave Hirsch fue un rabino que integró su perspectiva ética y religiosa con los principios del pragmatismo y la terapia ocupacional (Breines, 1992), trabajó con Jane Addams y Julia Lathrop colaborando en la fundación de la Sociedad de Artes y Oficios de Chicago. Además, participó en otras importantes transformaciones sociales, como la reforma judía de los Estados Unidos de América y también, siendo rabino de la Chicago Sinai Congregation, compartió el púlpito con Jane Addams apoyándola para ser la primera mujer que hablara en esa instancia (Breines, 1986). Hirsch creía fervientemente en el poder curativo de las ocupaciones, por lo que las empleaba en su trabajo; principalmente se orientó hacia los jóvenes desfavorecidos que delinquían y carecían de oportunidades sociales. Muchas de sus creencias eran coherentes con los principios cuáqueros de Addams (Breines, 1992).

Hirsch tuvo un rol importante en el desarrollo de la terapia ocupacional al organizar el curso de ocupaciones terapéuticas, junto a Julia Lathrop, para personas con invalidez en la Hull House (Morrison, 2016a). De este modo, Hirsch fue profesor de Eleanor Clarke Slagle y de Mary Potter Brooks Meyer (Breines, 1986), y junto a Addams, Lathrop y Graham Tylor, fundó la Escuela de Civica y Filantropía, y formó parte con Lathrop, de Chicago Charities Commission y la State Board of Charities (Addams, 1935/2004). Hirsch ejemplifica uno de los vínculos entre el pragmatismo y la joven terapia ocupacional, al igual que una figura bastante desconocida, Mary Potter Brooks Meyer. 
Brooks trabajó en Baltimore y en el Pathological Institute of the New York State Hospitals realizando visitas a los domicilios de los/as pacientes psiquiátricos y reportando sus hallazgos al equipo médico (John Hopkins Medicine, 2020). Ella estaba convencida de que los factores sociales afectaban la salud mental de las personas. Pero, donde destacó principalmente fue en el Worcester Massachusetts State Hospital, desarrollando un programa de ocupaciones terapéuticas. Este programa incluía aspectos de la psicobiología de Adolf Meyer (su esposo) y de las artes y oficios con fines terapéuticos. Realizó una sistematización similar a la de Slagle desde un enfoque pragmatista y fue una de las primeras trabajadoras sociales en ofrecer intervenciones sistemáticas para ayudar a pacientes, sus familias y al personal médico (Levine, 1987; Mansfield, 2013).

La Hull House ofreció programas innovadores que tuvieron grandes colaboradores como Dewey o Mead, y formó a muchas personas influyentes en la sociedad como Brooks y Meyer. Este matrimonio estuvo en contacto directo con Lathrop, quien invitó a Meyer a incorporarse a la Hull House. Por ello, Meyer tenía mucho aprecio a Lathrop, y ambos discutieron temas de interés en la salud mental y las reformas en los tratamientos de las/os pacientes psiquiátricos (Addams, 1935/2004).

En Chicago, la colaboración entre Meyer y Lathrop dio como resultado la aplicación de un programa empleando las artes y los oficios en pacientes crónicos. Lathrop había estudiado encuadernación en Kelmscott Press con William Morris y quiso mejorar las vidas de las personas empleando artes y oficios. Consiguió este objetivo influyendo en el Illinois State Board of Charities and Correction. Y junto a Hircsh, en 1906, organizó un programa de entrenamiento en ocupaciones terapéuticas para enfermeras (Levine, 1987).

El vínculo de Meyer con la terapia ocupacional se debe a su asociación con Slagle, Dunton, Lathrop y Addams (Addams, 1935/2004). Y aunque Dunton no compartía del todo el desarrollo teórico de Meyer, lo que se expresa en que rara vez colaboraron en encuentros a pesar de que trabajaron en el mismo hospital durante años, pertenecían a varias sociedades en común y tenían mutuo aprecio por su trabajo profesional (Breines, 1986).

Cuando Meyer asistía a Clark University and Worcester Insane Hospital en Massachusetts, conoció a William James que en ese entonces era catedrático de la Universidad de Harvard. También tuvo relación con George Herbert Mead, gracias a que ambos pertenecían casi a las mismas asociaciones en Chicago. Cuando Dewey y Mead estaban en la Universidad de Chicago, Meyer estaba en Kankakke, (Illinois), por lo que visitaba la Universidad (Breines, 1986).

La Hull House fue el punto de encuentro de grandes pensadoras/es y reformadores/as sociales, que combinaban el pragmatismo con el activismo social. Pero no fue solo allí donde se establecieron contactos con las y los principales exponentes. Por ejemplo, Dewey dejó Chicago en 1904 y Slagle ingresó en 1908 y, aunque no hay información que indique que ella estuviera antes en Chicago, de todos modos tomó clases con él en la Universidad de Columbia (Morrison, 2014).

Por otro lado, Susan Tracy fue seguidora de Dewey y usó muchas de sus ideas en su tratamiento ocupacional; al mismo tiempo mantuvo contacto con Dunton y se influenciarían mutuamente; en 1921 Tracy adoptaría el término "terapia de la ocupación”, concebido originalmente por Dunton, y él diseñaría programas de formación, basados en los escritos de Tracy (Bing, 1981); además Tracy hizo referencia a Dewey en su texto de 1910. Barton sería quien daría el nombre definitivo a la disciplina como terapia ocupacional desde las ideas iniciales de Tracy y Dunton 
(Breines, 1986). Por otro lado, Slagle tomó varios de los escritos de James como referencia de su entrenamiento en hábitos (Slagle, 1936).

\section{El Desarrollo Teórico de la Terapia Ocupacional y el Pragmatismo}

Susan Tracy, Susan Cox Johnson, Eleanor Clarke Slagle y otras fundadoras/es estuvieron en contacto directo con el pragmatismo y pusieron en práctica sus principales ideas lo que se habría expresado en sus escritos y tratamientos hacia sus pacientes, y también de manera implícita en sus programas formativos (Morrison, 2014).

Aunque aquí se ha considerado que el pragmatismo es una de las bases filosóficas de la terapia ocupacional, no ha sucedido lo mismo en varios escritos de la literatura disciplinar, ni en los programas formativos de terapeutas ocupacionales, como ha quedado en evidencia en diferentes publicaciones (Breines, 1986; Gordon, 2002; Hooper \& Wood, 2002; Ikiugu, 2001; Ikiugu \& Schultz, 2006; Morrison, 2014, 2016b, 2017).

Una de las explicaciones respecto a la ausencia del pragmatismo en el currículo de formación de terapia ocupacional es que, debido al androcentrismo muchos escritos de las mujeres fundadoras no fueron considerados al mismo nivel que los de los varones (Morrison, 2014). El ejemplo más claro de esto es que se considera que el primer documento formal de la disciplina es de Dunton (1919) o incluso Meyer (1976) en 1922, y no el de Tracy (1910).

Es curioso que a pesar de la mención explicita de Tracy a Dewey (Tracy, 1910), y la alusión a la teoría de los hábitos de pragmatistas como James y Peirce, a los que Slagle se refirió (Slagle, 1922, 1936, 1944), muchas y muchos terapeutas ocupacionales no han considerado al pragmatismo como una epistemología constituyente de la profesión. Aun cuando, eso se ha reflejado en elementos centrales como el holismo.

\section{El holismo en la terapia ocupacional}

Desde sus inicios, la perspectiva holística de la terapia ocupacional buscó comprender como las enfermedades que afectaban a una parte del cuerpo, repercutían tanto en el desempeño de ocupaciones como en la salud y bienestar de las personas (Morrison, 2017). Además, el holismo hace alusión a cómo los problemas "físicos" generan problemáticas en la mente (Gordon, 2002), por lo que se establece la relación psiquesoma, mente-cuerpo, en un todo integrado, y que además, esta dentro de un contexto particular. Como también lo describieron pragmatistas como Dewey (1922), cuando hicieron referencia a los hábitos y las ocupaciones.

Bajo la premisa anterior, desde los inicios de la profesión se evidenció cómo pacientes lograban mejorar de sus afecciones mentales y físicas cuando se ocupaban, por ejemplo, en artes y oficios (Slagle, 1922). Esto sirvió como justificación de la terapia ocupacional de principios del siglo XX, donde la perspectiva holística de la salud fue fundamental para la comprensión del ser humano (Bing, 1981). Así, los tratamientos e intervenciones ocupacionales fueron construidas intentando abolir las dicotomías que dividían al cuerpo de la mente, y buscaron complejizarse mostrando sus beneficios tanto en los procesos de recuperación como en la restauración de la funcionalidad física y la actitud mental (Quiroga, 1995). 
La mirada holística es propia de las y los terapeutas ocupacionales y posee una base en el pragmatismo (Wish-Baratz, 1989). Muchas veces esta mirada particular, ha generado algunas confusiones en la disciplina. Esto, debido a que se observa el hacer de la profesión, pero desde una mirada basada en dicotomías, por ello se tiende a confundir con otras disciplinas como la psicología o la fisioterapia, al "observarse" desempeños similares en la práctica.

Esta dificultad para comprender la disciplina por su holismo pragmatista, es un problema que proviene de la perspectiva de la ciencia neopositivista (Olivares et al., 2015). La ciencia como un procedimiento que permite, por medio de la observación, identificar resultados positivos en un fenómeno determinado fue una idea imperante durante el desarrollo del neopositivismo (Olivares et al., 2015). Esto, daría cabida a intentar comprender la terapia ocupacional, entendida como "[...] un tratamiento, que en sus orígenes se consideró una parte de la psicoterapia” (Gordon, 2009, p. 205), desde una manera objetiva y cuantificable.

Avalar teóricamente el concepto de ocupación trajo consigo algunas dificultades para las/os primeros terapeutas ocupacionales, específicamente para construir un cuerpo de conocimiento claro y coherente en el mundo científico (Kielhofner, 2009; Quiroga, 1995). Aunque, durante el primer tercio del siglo XX en los Estados Unidos de América, la terapia ocupacional contó con claras bases científicas ${ }^{1}$ (Morrison, 2014), el neopositivismo en la medicina, expresado en el modelo biomédico, entró a cuestionar los fundamentos epistemológicos de la terapia ocupacional, que no estaban del todo consolidados, generando que la disciplina estuviese bajo el ala de la medicina durante muchas décadas (Kielhofner, 2009). Lo anterior, fue una de las causas, también es importante considerar el rol de la guerra en ese proceso.

El modelo biomédico neopositivista se centraba en los resultados cuantificables de los/as pacientes, mientras que la terapia ocupacional en el proceso de recuperación que tenía una orientación más cualitativa y humanista (Quiroga, 1995). Este dilema entre lo científico neopositivista y lo humanista se mantendría durante gran parte del desarrollo de la disciplina, incluso influyendo en su desarrollo en Latinoamérica (Monzeli et al., 2019; Morrison et al., 2016). De todos modos, el holismo es una herencia pragmatista que cada vez logra comprenderse de mejor manera en el mundo de la medicina (Gaitán et al., 2019; Mezzich, 2007; Palma, 2004) y en, ciertos aspectos, la terapia ocupacional ha sido pionera en ello.

\section{La significación de la subjetividad de las personas}

Desde el pragmatismo, la terapia ocupacional comprende a los seres humanos involucrados completa y permanentemente en ocupaciones, donde cada desempeño es individual, y es percibido por las personas de forma única, transitoria y considerando los

\footnotetext{
${ }^{1}$ Por ejemplo, las publicaciones de Slagle sobre el entrenamiento en hábitos, en el proceso de reinserción social de personas con enfermedades crónicas, fue reconocido en gran parte de la psiquiatría como un método novedoso y altamente efectivo con una fundamentación científica. Las propuestas de Meyer sobre el equilibrio ocupacional desde la perspectiva de la psico-biología, también se consideraron como una base importante para los procesos de comprensión de la enfermedad de la época, donde el contexto tomaba un rol protagónico, no así los elementos reduccionistas que posteriormente, durante el segundo tercio del siglo XX, tomarían mayor protagonismo (Morrison, 2014).
} 
significados culturales (Slagle, 1934b). Además, establece que las ocupaciones ocurrenen un entramado complejo de relaciones transaccionales en el entorno (Mead, 1934).

De este modo, la primera generación de terapeutas ocupacionales construyó una mirada, además de holística, humanista donde la persona es el centro. Por ende, brinda de importancia las características particulares, a la vez que las habilidades, valores, problemas, necesidades y herencia cultural específicas de cada persona (Finlay, 2001).

Para comprender la aparente simpleza de lo anterior, es necesario profundizar en cómo el pragmatismo discutía estos temas.

Lo que entendemos por realidad es una percepción individual, al mismo tiempo que colectiva, y única (Putnam, 1999). Esto quiere decir, que frente a la pregunta: cómo conocemos el mundo, el pragmatismo propondrá que la realidad es un consenso social, transitorio, parcial y cuya utilidad debe ser evaluada de forma permanente (Addams, 1902/2002; Dewey, 1922; Mead, 1934).

Esta percepción de la realidad, está construida en base a ideas. Las ideas representan un punto central en la filosofía pragmatista, pues permiten establecer consensos sociales y mantener o transforma la misma realidad. Así, las ideas dependen de un contexto específico y son alterables en función de las interacciones que desarrollen en esos contextos (incluyendo la interacción con otras personas). A una idea "le ocurre la verdad", esto quiere decir que no posee veracidad a priori, su valoración y consideración como verdadera, dependerá de las condiciones donde se encuentre. Así, su futuro es una probabilidad (James, 1892, 1909/1974).

De lo anterior es que las ideas en las personas, constituidas por sus experiencias, juicios y percepciones, dan cuenta de sus historias de vida y deseos. Esto representa a una de las ideas fundacionales en la formación de terapeutas ocupacionales: que la situación de una persona es una realidad, es $s u$ realidad, por ende, conforma un valor independiente de las opiniones médicas u profesionales, aunque estás últimas sean "verdades científicas". Esto podría traducirse en diferentes situaciones, por ejemplo, que la opinión de un/a paciente es crucial en la decisión frente a un tratamiento médico o terapéutico que le atañe.

Aunque la realidad sea un consenso y pueda ser transformada en la medida que nos ponemos de acuerdo en tono a nuestras ideas, es relevante mencionar que, lamentablemente, no todas las personas participan en igualdad de condiciones para vivir y pensar esa realidad (Addams, 1902/2002; Dewey, 1916/2004). Para Addams y Dewey la educación era una forma de equilibrar la balanza para el desarrollo de una reflexión crítica para repensar la realidad, lo que tomaría la primera generación de terapeutas ocupacionales dentro de los procesos de intervención profesional.

Personas mutiladas, dentro de hospitales psiquiátricos, viviendo en la calle, etc. no participan en aquel consenso de la realidad ni menos en los procesos que lograsen revertir las condiciones sociales que reproducían las desigualdades sociales. Así, las personas aprendían que su realidad no es posible de ser modificada. Por ello, las tempranas intervenciones de terapia ocupacional intentaron, por medio de la ocupación, generar ambientes enriquecidos para estas personas excluidas, de manera de brindar estímulos que desarrollaran sus habilidades y mejorasen sus situaciones de salud, al mismo tiempo que buscaron generar cambios en algunas de las estructuras que perpetuaban los procesos de exclusión. 
De este modo, comprender la subjetividad y el lugar en el mundo de una persona permitiría repensar ambos elementos y cuestionar lo dado por realidad, al mismo tiempo que facilitar procesos de inclusión. Esto se enraíza en la creencia de que sí es posible transformar una realidad dada.

Otra expresión de la significación de la subjetividad de cada persona, tiene relación el tipo y diseño de los tratamientos empleados en la terapia ocupacional. Comprendiendo que la verdad es transitoria, y por ende todas sus expresiones como tratamientos médicos, psiquiátricos, etc., para la temprana terapia ocupacional, la estandarización de los medios terapéuticos no era un objetivo. En cambio, como lo propusieron diferentes mujeres interventoras (Addams, 1935/2004; Johnson, 1919; Slagle, 1922, 1931; Tracy, 1910) no son las personas quienes deben adaptarse a los tratamientos o medios de intervención, si no al revés.

$Y$ en el proceso anterior, era necesario tomar conciencia de las propias habilidades y formas de relación y orientarlas hacia el trato digno y amable, ya que el rol del/a terapeuta ocupacional era fundamental en el proceso exitoso de un tratamiento y en la mejora del estado de salud y bienestar de las personas (Slagle, 1922, 1934a). Esto, reforzado bajo la idea del pragmatismo de que las personas se constituyen por medio de la interacción con el contexto y en la relación con las demás personas (Mead, 1934, 1936). Así, se buscó zanjar las privaciones del entorno en los procesos de intervención.

La significación por la subjetividad y la mirada holística son solo dos de las diferentes herencias del pragmatismo en la terapia ocupacional. Como parte de la diversidad de influencias, se dará cuenta de un aspecto más, esto es relacionado con el desarrollo teórico de Adolf Meyer en el mundo médico.

\section{El pragmatismo de Meyer y la terapia ocupacional}

Meyer no fue solo una figura importante para la terapia ocupacional. Se le ha reconocido como uno de los que contribuyeron a cambiar el concepto de psiquiatría en los Estados Unidos de América al trabajar en los aspectos de socialización y de la vida personal de sus pacientes (Marx, 1993). Sin embargo, en opinión de historiadores de la terapia ocupacional como Gordon (2002), considerarlo como "el gran líder teórico" de la época es exagerado, debido a que muchos de sus postulados son reconstrucciones del pragmatismo. Empleó términos como: adaptación, acción, evolución, sistemas integrados, temporalidad, realidad a través de la transacción, bienestar social, salud a través de la educación, entre otros (Meyer, 1976). Influyó en patología médica, neurología, psiquiatría, administración, educación médica e higiene mental (Breines, 1986), además de contribuir al trabajo social, la medicina y la enfermería (Addams, 1935/2004).

Respecto a sus bases pragmatistas, Meyer estaba de acuerdo con Peirce (1904/2004) en atacar las dicotomías cartesianas para explicar el mundo y en comprender que el ser humano es parte de la naturaleza y que se encuentra constantemente en interacción con el ambiente; al mismo tiempo, los órganos sensitivos de las personas se desarrollan de acuerdo a la adaptación vital para la supervivencia. Consideró que la mente no es un ente abstracto, ni separado del cuerpo y, como Peirce, no concebía que el pensamiento estuviese separado de las circunstancias, la mente crea soluciones a problemas de la experiencia. Por esto, Meyer concluyó que la manera de erradicar la antigua disputa 
entre los términos fisiológicos y psicológicos sobre el ser humano era dejar de pensar en dicotomías (Lidz, 1985).

Otro ejemplo es lo señalado por James y Peirce sobre los hábitos y la salud. Ya, muchos médicos de la época consideraban que los estilos de vida generados por la industrialización eran uno de los principales causantes de enfermedades típicas como la neurastenia (Addams, 1935/2004; Gordon, 2002).

Particularmente, James propuso explicaciones sobre las enfermedades mentales. Señaló que estas tenían un aspecto social importante y que no solo era provocado por agentes biológicos (James, 1985). De aquí que Meyer también postulara que era necesario revisar las historias de vida de sus pacientes, ya que no eran seres aislados del mundo (Lidz, 1985; Marx, 1993). Este último punto fue uno de sus principales aportes a la psiquiatría: centrarse en la vida de sus pacientes, en sus experiencias y decisiones previas, observar sus circunstancias, y evaluar cómo ellas fueron insumos para la enfermedad mental.

Estos planteamientos fueron consolidados en su estancia en la Hull House, al observar el trabajo de Addams y Lathrop. Ellas consideraban que las condiciones sociales tienen un rol importante en la salud mental (Addams, 1902/2002, 1912), por lo que explorar estos aspectos podría dar alguna idea de cómo realizar un tratamiento más certero.

La vida de las personas y el "sentido común" era algo que Meyer devolvería a la psiquiatría, que había comenzado a "[...] buscar causas en las células del cerebro, en fisiología o bioquímica” (Lidz, 1985, p. 43). De este modo, además de las ideas de Peirce, James, Addams y Lathrop, consideró los conceptos de temporalidad de Mead (1934, 1936), quien concibió al ser humano como una persona temporal y construida desde influencias subjetivas de sí mismo y otros/as.

Así, Meyer pensó que no puede explicarse una enfermedad mental si no se considera la historia de vida de las personas (Lidz, 1985), por lo que señaló que en el hacer, la acción y la experiencia se constituye el ser humano como un ser social (Breines, 1986).

Por todo lo anterior, en el hacer es donde existían mayores oportunidades para mejorar a las y los pacientes. El equilibrio en los estilos de vida, la importancia de los hábitos, y el desarrollo de la salud a través de actividades fueron elementos centrales de las teorías pragmatistas que Meyer compartió con la temprana terapia ocupacional (Gordon, 2002). Estos elementos fueron desarrollados por esta primera generación de profesionales bajo la idea de ocupación. Para Meyer: “[...] la ocupación es, con una buena dirección, el lado más esencial del tratamiento higiénico para el/a más enfermo/a de los/as pacientes"2 (como se cita en Bing (1981, p. 511), debido a que en la ocupación se integra todo el cuerpo en acción. Y bajo una dirección terapéutica, es posible desarrollar los aspectos "dormidos" de la salud de las/os pacientes.

Meyer postuló que la terapia ocupacional concibe la unidad mente-cuerpo en un todo con el ambiente. De este modo, el problema de las personas con enfermedad mental es un problema de adaptación a su contexto y la ocupación es el mejor tratamiento para esos problemas (Meyer, 1976).

Las ideas de hábitos y transacción con el ambiente, permiten considerar las actividades como instrumentos de interacción que han permitido la supervivencia y el

${ }^{2}$ Traducción propia. 
progreso (James, 1985). Al igual que postula el pragmatismo, Meyer estaba convencido de que los seres humanos pueden transformarse a sí mismos. Nuestras acciones dependen de nuestras decisiones y las decisiones se toman empleando un juicio crítico (Lidz, 1985). Estas ideas se integraron en el desarrollo de su psicobiología, y su perspectiva holística sobre el proceso salud-enfermedad.

Finalmente, algunos de los elementos que desarrolló en la terapia ocupacional fueron que el comportamiento humano se integra en la mente y lo que una persona piensa afecta sus funciones vitales, desde un nivel celular hasta su simbolización, afectando sus relaciones sociales. Los niveles físico, químico, celular, psicológico son tremendamente relevantes para ayudar a una persona, pero de manera independiente, por separado, no dan cuenta de lo que la persona es (Lidz, 1985).

\section{Ideas Finales}

Este artículo ha buscado evidenciar las relaciones entre el pragmatismo y la temprana terapia ocupacional en los Estados Unidos de América. Claramente se observa una relación de influencia en los ideales, teorías y prácticas de la profesión, con los planteamientos de pragmatistas clásicos como William James, Charles Peirce, George Mead, John Dewey y Jane Addams. Comprender al pragmatismo es acercarse más a la comprensión de la propia terapia ocupacional.

Como desafíos, sería interesante discutir qué aspectos del pragmatismo son coherentes con las formas de hacer terapia ocupacional y ciencia ocupacional en Latinoamérica. ¿Es una epistemología que nos sirve para pensar a la ocupación hoy? ¿Es un aporte en las prácticas de la terapia ocupacional? ¿Es posible integrar las perspectivas pragmatistas a los enfoques Latinoamericanos de la profesión? Estas cuestiones quedan como parte del debate abierto y, ciertamente, sería una contribución que diferentes colegas en Latinoamérica pudiesen discutir al respecto.

\section{Referencias}

Addams, J. (1912). Twenty years at Hull House with autobiographical notes. New York: The McMillan Company.

Addams, J. (2002). Democracy and social ethics. Illinois: University of Illinois Press. (Original work published 1902).

Addams, J. (2004). My Friend, Julia Lathrop. New York: Macmillan. (Original work published 1935).

Bing, R. K. (1981). Occupational therapy revisited: a paraphrastic journey. The American Journal of Occupational Therapy, 35(8), 499-518.

Breines, E. (1986). Origins and adaptations: a philosophy of practice. New Jersey: Geri-Rehab.

Breines, E. (1992). Rabbi Emil Gustave Hirsch: ethical philosopher and founding figure of occupational therapy. IJOT: Israeli Journal of Occupational Therapy, 1(1), 9.

Dewey, J. (1922). Human nature and conduct: an introduction to social psychology. New York: Modern Library.

Dewey, J. (2004). Democracia y educación. Madrid: Morata. (Original work published 1916).

Dunton, W. R. (1919). Reconstruction therapy. Philadelphia: Saunders.

Finlay, L. (2001). Holism in occupational therapy: elusive fiction and ambivalent struggle. The American Journal of Occupational Therapy, 55(3), 268-276. 
Gaitán, A., Fontana, A., \& Vicco, M. (2019). El coexistir del mecanicismo reduccionista y el holismo. Revista Argentina de Medicina, 7(4), 224-227.

Gordon, D. (2002). Therapeutics and science in the history of occupational therapy (PhD dissertation). University of Southern California, Los Angeles.

Gordon, D. (2009). The history of occupational therapy. In E. B. Crepeau, E. S. Cohn \& B. B. Schell (Eds.), Willard and Spackman's occupational therapy (pp. 203-215). Philadelphia: Lippincott Williams \& Wilkins.

Hooper, B., \& Wood, W. (2002). Pragmatism and structuralism in occupational therapy: the long conversation. The American Journal of Occupational Therapy, 56(1), 40-50.

Ikiugu, M. N. (2001). The philosophy and culture of occupational therapy (PhD dissertation). Texas Woman's University, Texas.

Ikiugu, M. N., \& Schultz, S. (2006). An argument for pragmatism as a foundational philosophy of occupational therapy. Canadian Journal of Occupational Therapy, 73(2), 86-97.

James, W. (1892). The will to believe. New York: Longmans, Green, and Co.

James, W. (1974). El significado de la verdad. Buenos Aires: S.A. de Ediciones. (Original work published 1909).

James, W. (1985). Habit. Its importance for psychology. Occupational Therapy in Mental Health, 5(3), 55-67. (Original work published 1892).

John Hopkins Medicine. (2020). Psychiatry and behavioral sciences: the history of psychiatry social work. Johns Hopkins Medicine. Recuperado el 29 de agosto de 2020, de https://www.hopkinsmedicine.org/psychiatry/expert_team/social_work/dept_history.html

Johnson, S. C. (1919). Occupational therapy, vocational reeducation and industrial rehabilitation. Modern Hospital, 12, 221-223.

Kielhofner, G. (2009). Conceptual foundations of occupational therapy practice. Philadelphia: FA Davis.

Levine, R. (1987). The influence of the arts-and-crafts movement on the professional status of occupational therapy. The American Journal of Occupational Therapy, 4(4), 248-254.

Lidz, T. (1985). Adolf Meyer and the development of American Psychiatry. In K. D. Serrett (Ed.), Philosophical and historical roots of occupational therapy (pp. 33-54). New York: Harworth Press.

Mansfield, J. (2013). Recreational therapy-1880s to 1939. Recuperado el 29 de agosto de 2020, de https://www.recreationtherapy.com/history/rthistory2.htm

Marx, O. (1993). Conversation Piece: Adolf Meyer and psychiatric training at the Phipps Clinic an interview with Theodore Lidz. History of Psychiatry, 4, 245-269.

Mead, G. H. (1934). Mind self and society from the standpoint of a social behaviorist. Chicago: University of Chicago.

Mead, G. H. (1936). Movements of thought in the nineteenth century. Chicago: University of Chicago.

Meyer, A. (1976). The philosophy of occupation therapy. Reprinted from the Archives of Occupational Therapy, volume 1, pp. 1-10, 1922. The American Journal of Occupational Therapy, 31(10), 639642.

Mezzich, J. E. (2007). Psiquiatría orientada a la persona: articulación de la ciencia y el humanismo de la medicina. World Psychiatry, 5(2), 65-67.

Monzeli, G., Morrison, R., \& Lopes, R. E. (2019). Histórias da terapia ocupacional na América Latina: a primeira década de criação dos programas de formaçáo profissional. Cadernos Brasileiros de Terapia Ocupacional, 27(2), 235-250. http://dx.doi.org/10.4322/2526-8910.ctoao1631.

Morrison, R. (2014). La filosofia pragmatista en la terapia ocupacional de Eleanor Clarke Slagle: antecedentes epistemológicos e históricos desde los estudios feministas sobre la ciencia (PhD dissertation). Universidad de Salamanca, España.

Morrison, R. (2016a). Los comienzos de la terapia ocupacional en Estados Unidos: una perspectiva feminista desde los estudios de Ciencia, Tecnología y Género (siglos XIX y XX). Historia Critica, 62, 97-117. http://dx.doi.org/10.7440/histcrit62.2016.05. 
Morrison, R. (2016b). Pragmatist Epistemology and Jane Addams: fundamental concepts for the social paradigm of occupational therapy. Occupational Therapy International, 23(4), 295-304. http://dx.doi.org/10.1002/oti.1430.

Morrison, R. (2017). Terapia ocupacional y pragmatismo: contribuciones teóricas para la práctica. Santiago: Editorial Universitaria.

Morrison, R., Olivares, D., Graus, J. M., Cifuentes, D., Macari, C., Rojas, E., \& Parraguez, M. (2016). Silvia Gómez Lillo y cincuenta años de terapia ocupacional en Chile: una biografía. Revista Terapia Ocupacional Galicia, 13(24), 1-24.

Olivares, D., Morrison, R., Yañez, R., \& Carrasco, J. (2015). ¿Por qué puede ser difícil comprender qué es la terapia ocupacional? Una propuesta teórica desde cuatro perspectivas. Revista Chilena de Terapia Ocupacional, 15(1), 123-134. http://dx.doi.org/10.5354/0719-5346.2015.37136.

Palma, R. (2004). ¿El regreso del holismo? Revista Médica Clinica Las Condes, 15(3), 119-120.

Peirce, C. S. (2004). ¿Qué es el pragmatismo? The Monist /Grupo de estudios Peircianos de la Universidad de Navarra, 15(2), 161-181. (Original work published 1904)

Putnam, H. (1999). El pragmatismo: Un debate abierto. Gedisa.

Quiroga, V. (1995). Occupational therapy: the first thirty years, 1900-1930. Bethesda: American Occupational Therapy Association.

Reed, K. L. (2017). Identification of the people and critique of the ideas in meyer's philosophy of occupation therapy. Occupational Therapy in Mental Health, 33(2), 107-128.

Reed, K. L. (2018). Henry B. Favill and the School of Occupations: origins of occupational therapy practice and education. Occupational Therapy in Health Care, 33(2), 159-180.

Slagle, E. C. (1922). Training aides for mental patients. The American Journal of Occupational Therapy, 1(1), 11-18.

Slagle, E. C. (1931). The training of occupational therapist. The Psychiatric Quarterly, 5(1), 12-20.

Slagle, E. C. (1934a). The occupational therapy programme in the State of New York. The Journal of Mental Science, 80(331), 639-649.

Slagle, E. C. (1934b). Occupational therapy: recent methods and advances in the United States. American Journal of Physical Medicine \& Rehabilitation, 13(5), 289-298.

Slagle, E. C. (1936). The past, present and future of occupational therapy in the State Department of Mental Hygiene. The Psychiatric Quarterly, 10(1), 144-156.

Slagle, E. C. (1944). Syllabus for training of nurses in occupational therapy. New York: Department of Mental Hygiene.

Tracy, S. (1910). Studies in invalid occupation: a manual for nurses and attendants. Boston: Whitcome \& Barrow.

Wish-Baratz, S. (1989). Bird T. Baldwin: a holistic scientist in occupational therapy's history. The American Journal of Occupational Therapy, 43(4), 257-260. http://dx.doi.org/10.5014/ajot.43.4.257.

\section{Fuente de Financiamiento}

Departamento de Terapia Ocupacional y Ciencia de la

Ocupación de la Universidad de Chile, Chile.

\section{Autor para la correspondencia}

Rodolfo Morrison

e-mail: rodolfo.morrison@uchile.cl

\section{Editora}

Profa. Dra. Daniela Tavares Gontijo 